Supplementary Information for

\title{
Thermodynamic stability and aggregation kinetics of EF helix and EF loop variants of transthyretin
}

\author{
James A. Ferguson ${ }^{\dagger}$, Xun Sun $^{\dagger}$, H. Jane Dyson ${ }^{\dagger}$, Peter E. Wright ${ }^{*,+,}$ \\ ${ }^{\dagger}$ Department of Integrative Structural and Computational Biology and ${ }^{\ddagger}$ The Skaggs Institute of \\ Chemical Biology, The Scripps Research Institute, 10550 North Torrey Pines Road, La Jolla, \\ California 92037, USA \\ *Correspondence to Peter E. Wright: wright@scripps.edu
}



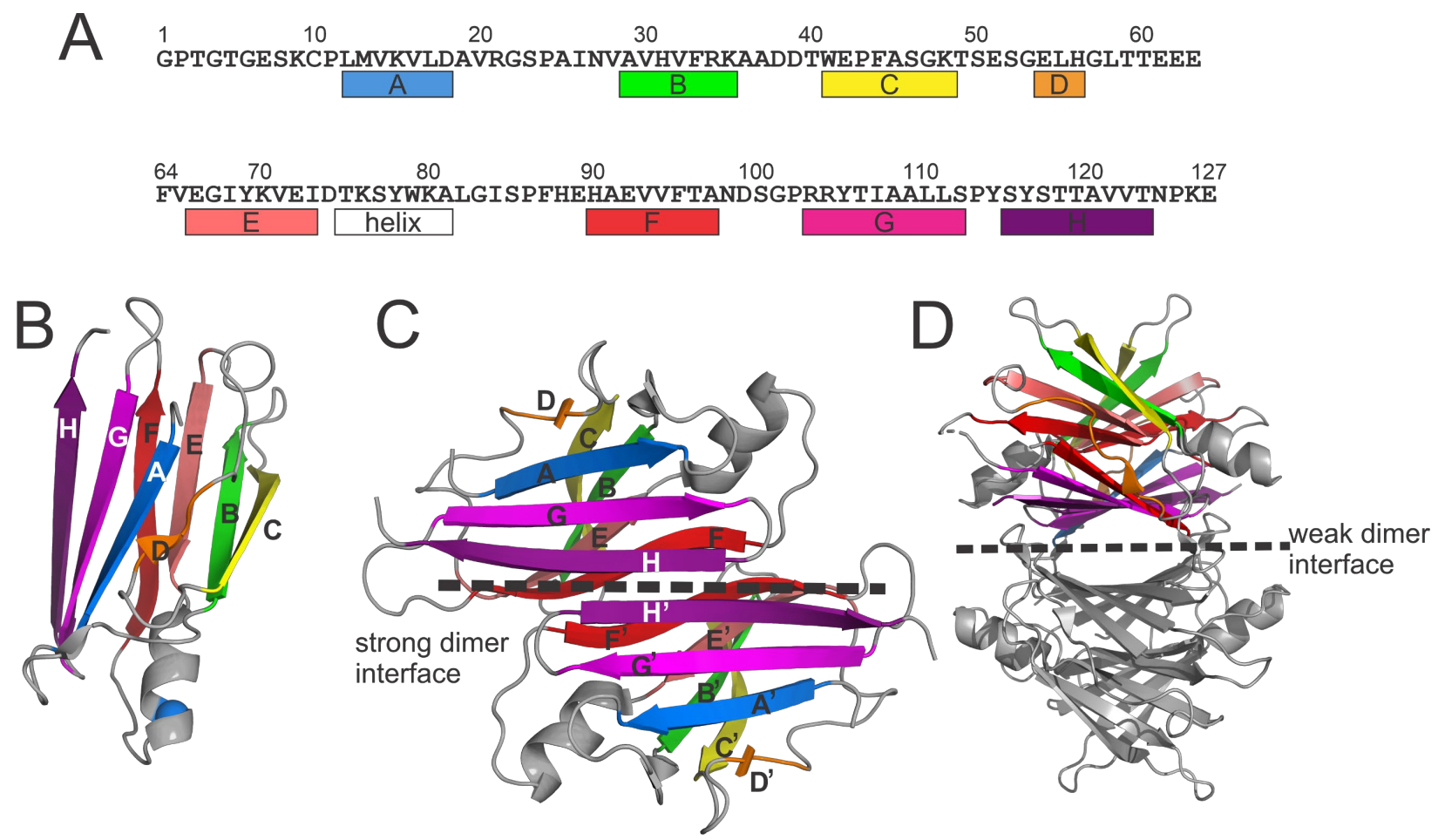

Figure S1. Amino acid sequence and structure of transthyretin (TTR). A. Amino acid sequence of the monomer unit of human transthyretin (excluding the signal peptide sequence), showing the positions of the $\beta$-strands $A-H$ and the helix between strands $E$ and $F$. B. Structure of a monomer unit of human TTR (PDB 2ROX ${ }^{1}$ ) showing the arrangement of the $\beta$-strands and the position of the C $\alpha$ of $K 80$ (blue sphere) towards the end of the helix. C. Dimer of TTR (PDB $2 \mathrm{ROX})$ showing the arrangement of $\beta$-strands across the strong dimer interface. Strands from the two monomer units are distinguished by the addition of prime ('). D. Tetramer of TTR (PDB $2 \mathrm{ROX}$ ) showing the location of the weak dimer interface. 


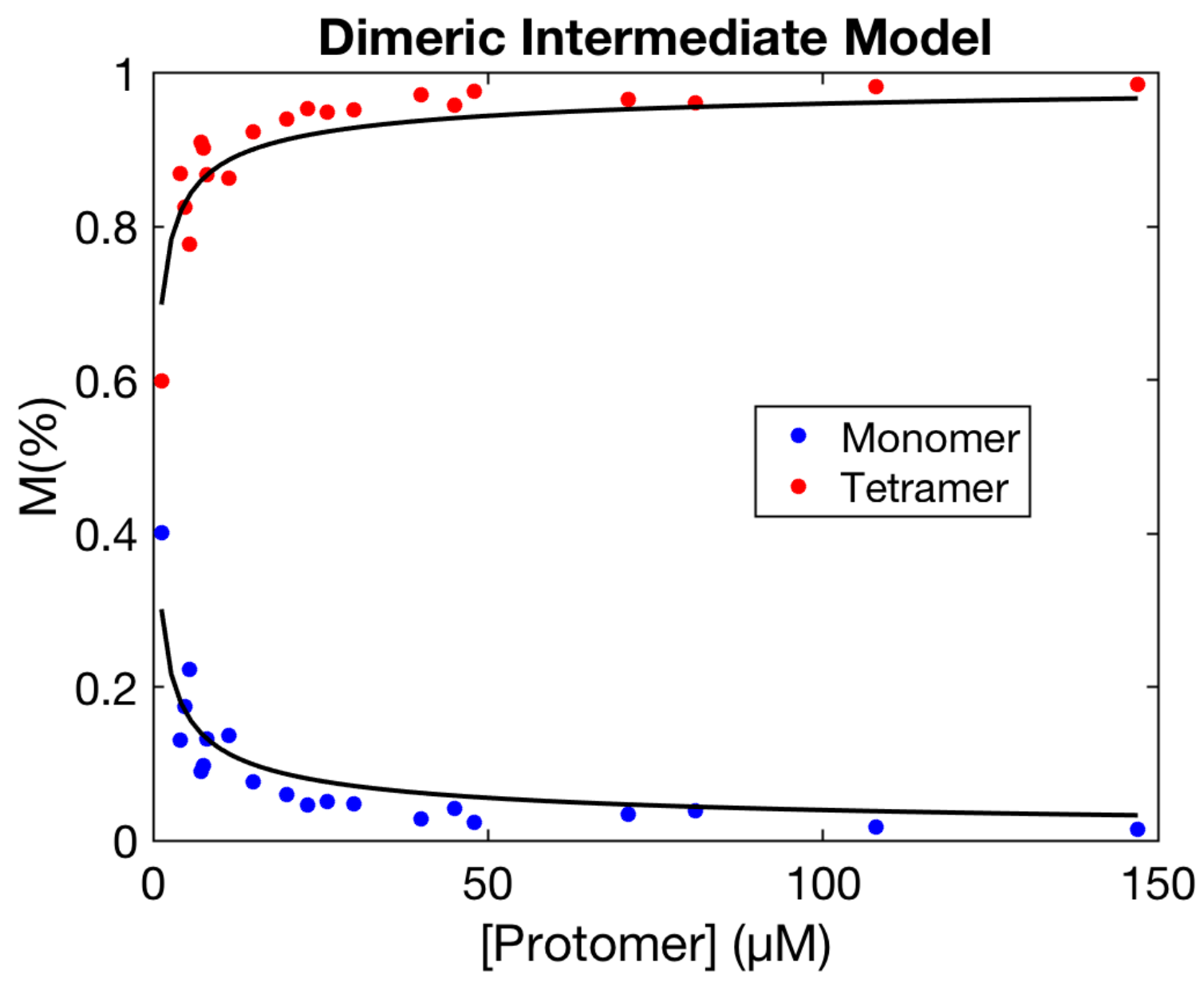

$$
\begin{gathered}
\mathrm{T} \stackrel{K_{\mathrm{d}}}{\longleftarrow} 2 \mathrm{D} \quad[\mathrm{D}]=\frac{1}{2}[\mathrm{M}] \\
M \%=\frac{\sqrt{K_{\mathrm{d}}^{2}+4 K_{\mathrm{d}} c_{\mathrm{t}}}-K_{\mathrm{d}}}{2 c_{\mathrm{t}}} \\
K_{\mathrm{d}} \sim 0.2 \mu \mathrm{M}
\end{gathered}
$$

Figure S2: This model assumes that the tetramer dissociates into a dimer which instantly dissociates to monomer, i.e. there is no dimer in solution. The model overestimates the amount of tetramer in the low concentration range and underestimates it in the medium concentration range. Sample data shown for $\mathrm{K}^{\circ} 0 \mathrm{D}^{\mathrm{F}}$ using NMR buffer with $100 \mathrm{mM} \mathrm{KCl}$ at $298 \mathrm{~K}$. 

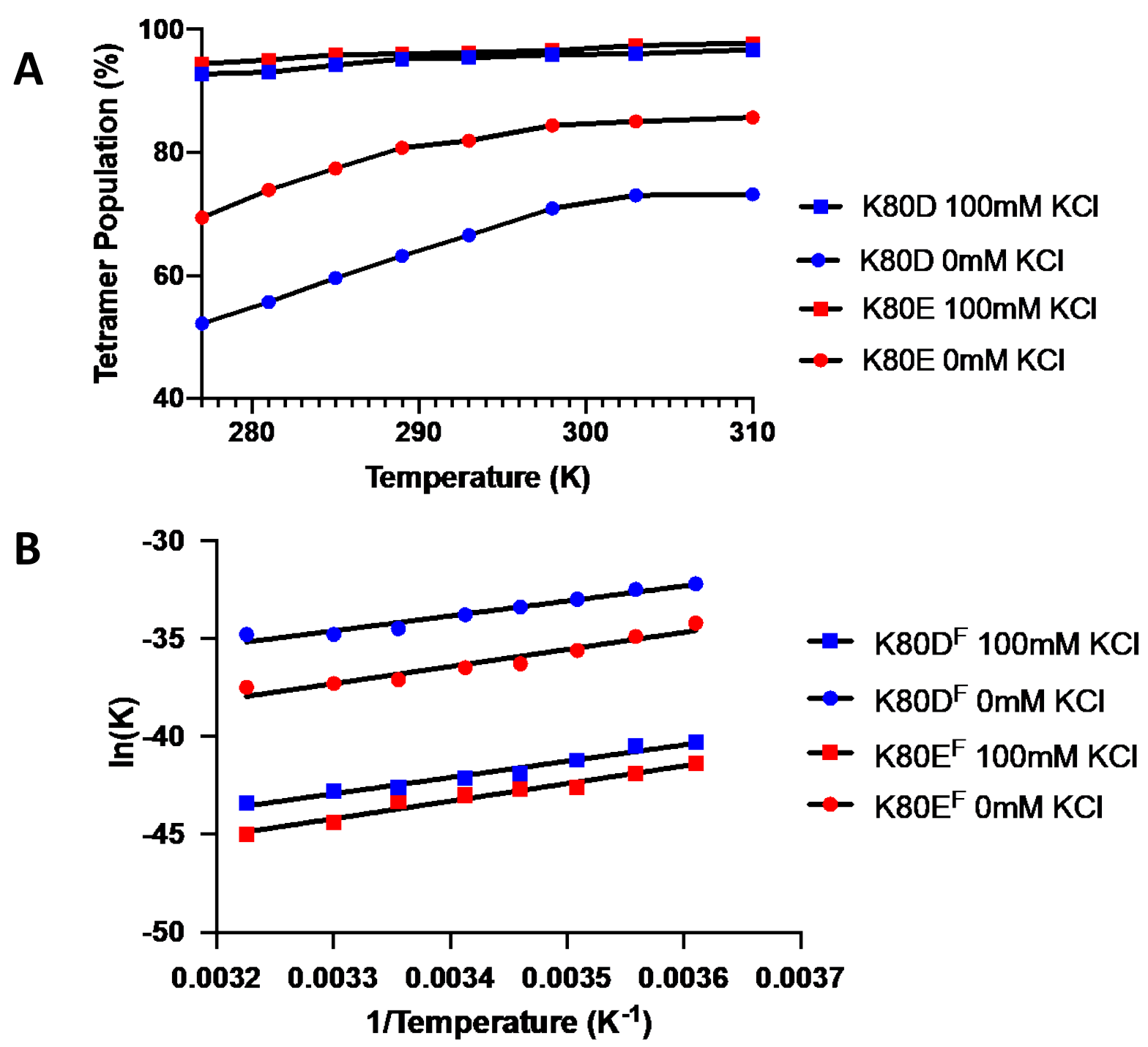

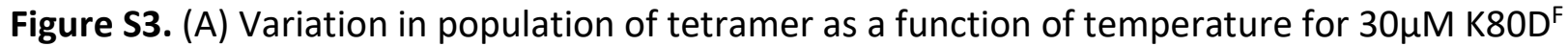
and $\mathrm{K} 80 \mathrm{E}^{\mathrm{F}}$ in $\mathrm{NMR}$ buffer in the presence and absence of $\mathrm{KCl}$ at $\mathrm{pH} 7.0$. Under all conditions, higher temperature favors the monomer over this temperature range.

(B) Van 't Hoff Plot. The equilibrium constant $\left(K=[M]^{4} /[T]\right)$ describing dissociation of the tetramer was determined from the relative populations of the tetramer and monomer at each temperature. 


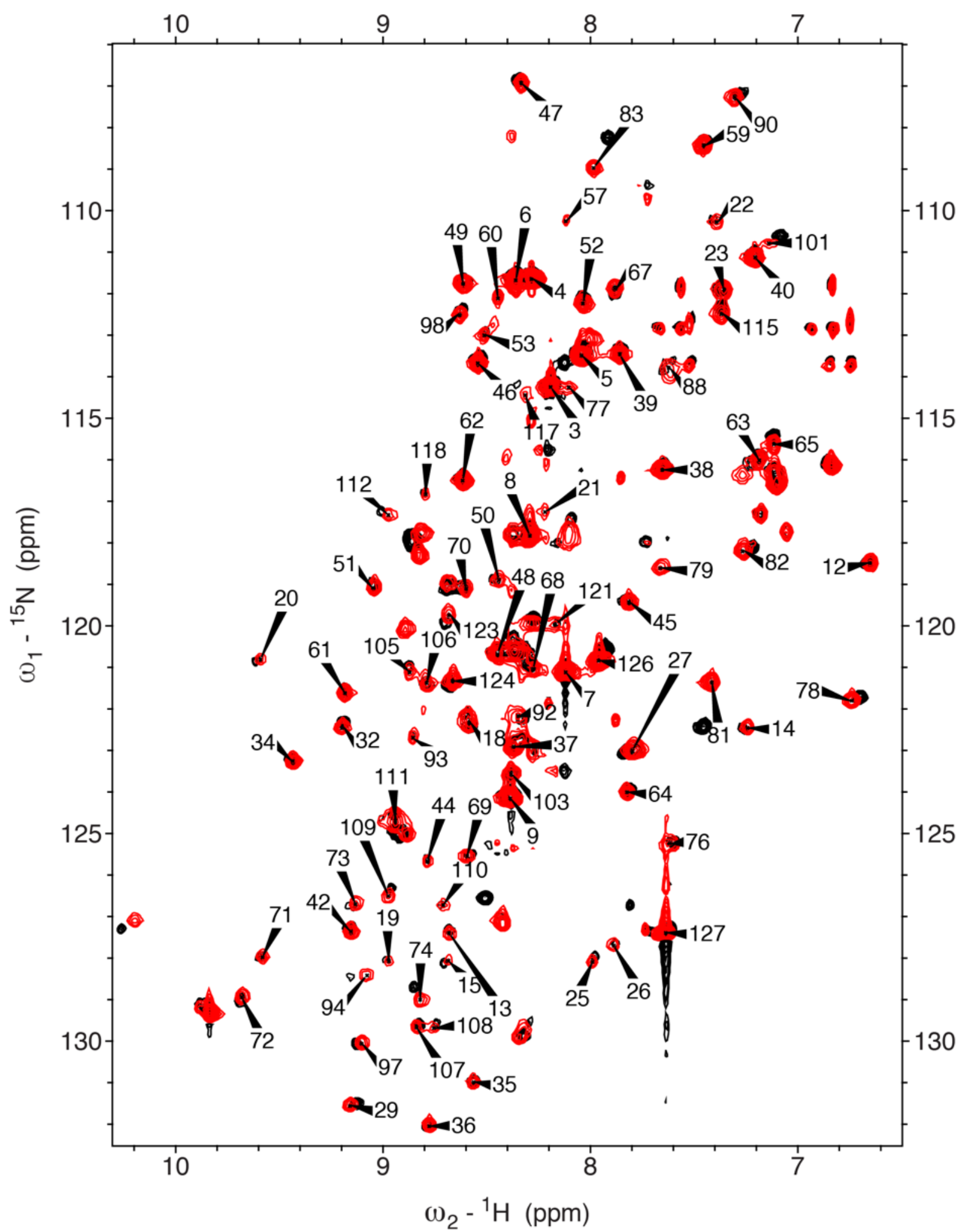

Figure S4: $\left[{ }^{1} \mathrm{H}^{15} \mathrm{~N}\right]-$ TROSY HSQC of K80D ${ }^{\mathrm{F}}$ (red) collected in NMR buffer at $298 \mathrm{~K}$ on a Bruker Avance 900 spectrometer. Overlaid on a $\left[{ }^{1} \mathrm{H}^{15} \mathrm{~N}\right]$-TROSY HSQC of TTR (black). Numbers indicate assigned ${ }^{1} \mathrm{H},{ }^{15} \mathrm{~N}$ cross peaks for the corresponding residues. 


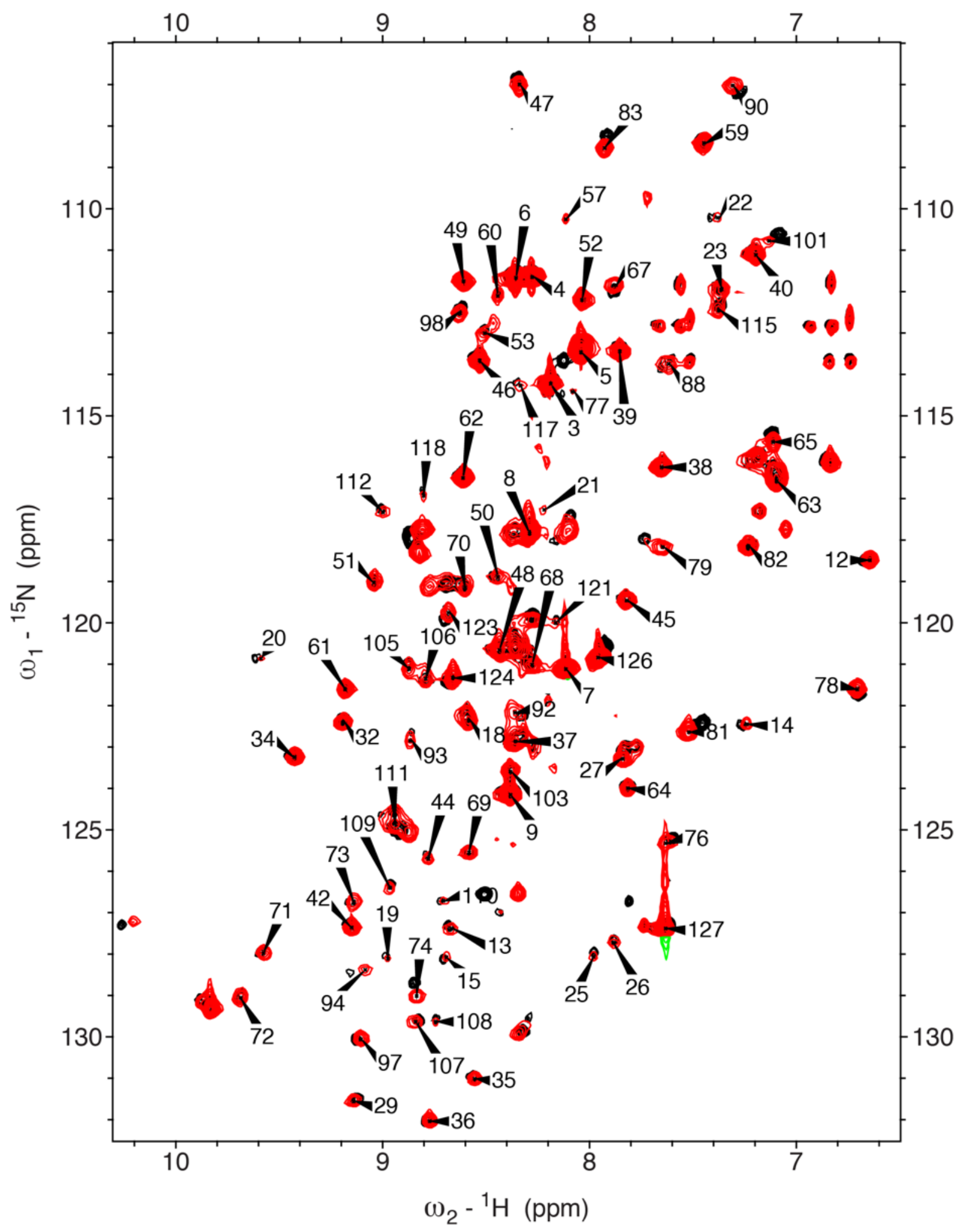

Figure S5: $\left[{ }^{1}{ }^{15} \mathrm{~N}\right]$-TROSY HSQC of K80EF (red) collected in NMR buffer at $298 \mathrm{~K}$ on a Bruker Avance 900 spectrometer. Overlaid on a $\left[{ }^{1} \mathrm{H}^{15} \mathrm{~N}\right]$-TROSY HSQC of TTR ${ }^{\mathrm{F}}$ (black). Numbers indicate assigned ${ }^{1} \mathrm{H},{ }^{15} \mathrm{~N}$ cross peaks for the corresponding residues. 

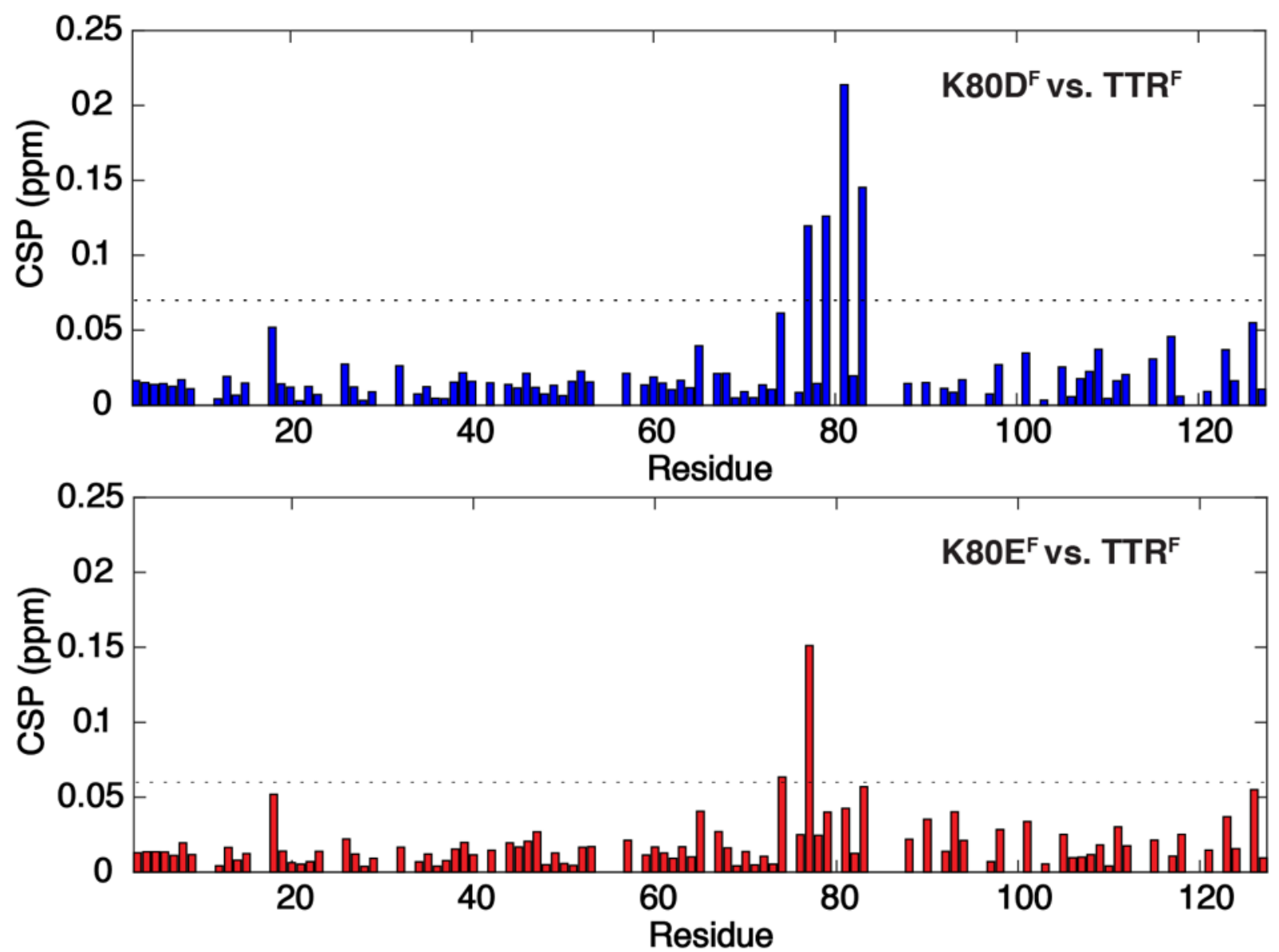

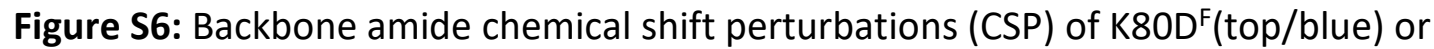
$\mathrm{K} 80 \mathrm{E}^{\mathrm{F}}$ (bottom/red) vs. TTR $\mathrm{T}^{\mathrm{F}}{ }^{1} \mathrm{H}^{15} \mathrm{~N}-\mathrm{HSQC}$ spectra were recorded in NMR buffer at $\mathrm{pH} 7.0$ and 298K. The dashed line denotes the mean plus one standard deviation. Weighted average backbone amide CSP values were calculated using $\sqrt{\left(\Delta^{1} \mathrm{H}\right)^{2}+\left(\Delta^{15} \mathrm{~N} / 5\right)^{2}}$. The HSQC spectra are shown in supplemental Figures S4 and S5. Chemical shifts of the parent TTR ${ }^{\mathrm{F}}$ (C10S-S85CBTFA) taken from ${ }^{2}$. 
A
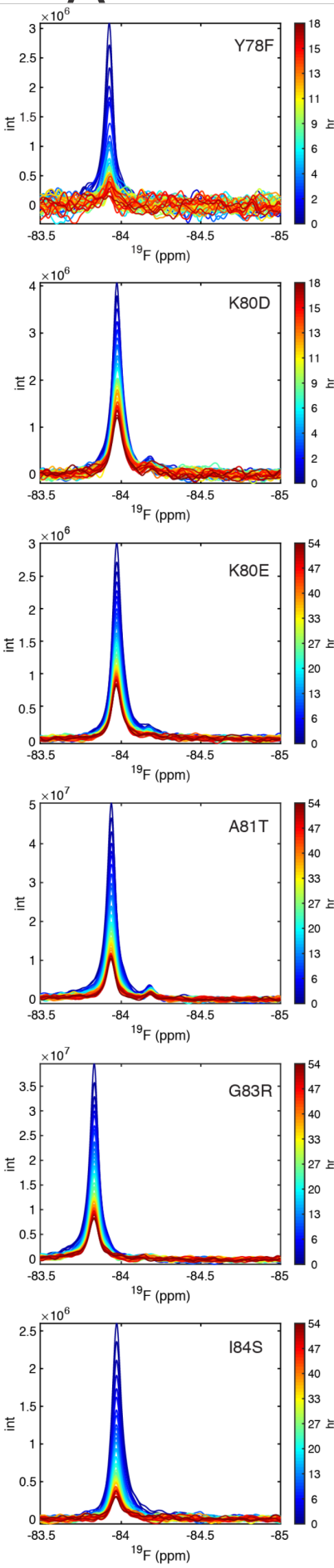
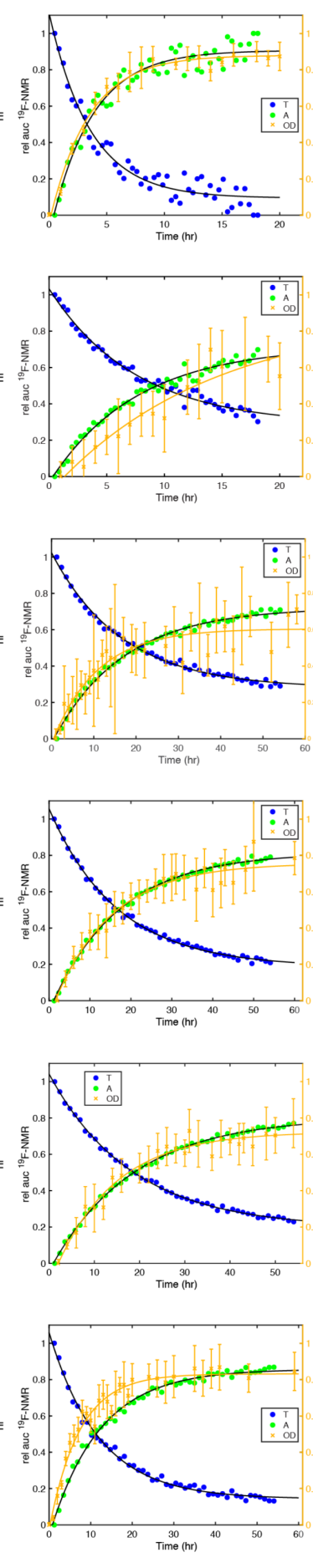
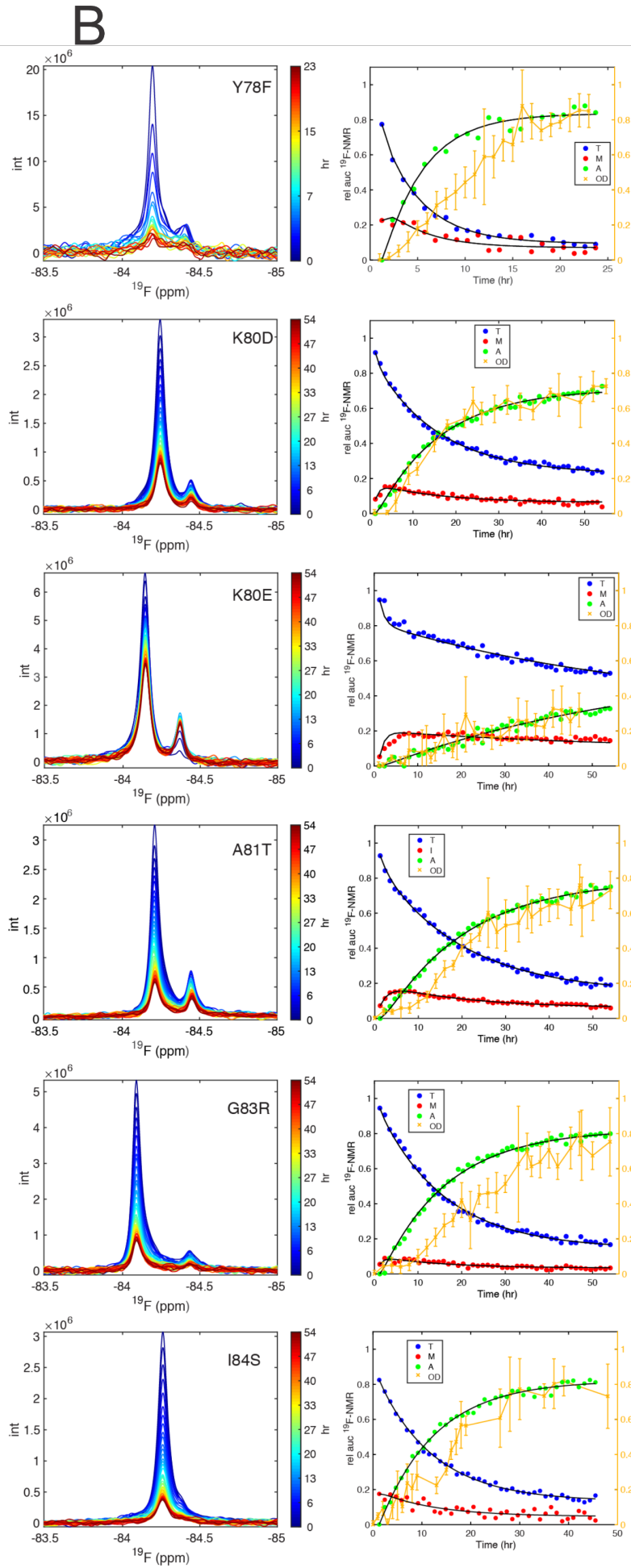

Figure S7. Monitoring aggregation kinetics of $T T R^{\mathrm{F}}$ mutants at $\mathrm{pH} 4.4(\mathrm{~A})$ at $310 \mathrm{~K}$ and $(\mathrm{B})$ at $298 \mathrm{~K}$, using ${ }^{19} \mathrm{~F}-\mathrm{NMR}$ and $\mathrm{OD}_{330}$ measurements. The left panels for both $(\mathrm{A})$ and $(\mathrm{B})$ show the overlaid time series of ${ }^{19} \mathrm{~F}-\mathrm{NMR}$ spectra with raw peak intensities. The right panels show normalized ${ }^{19} \mathrm{~F}$-NMR peak areas and normalized $\mathrm{OD}_{330}$ data. The ${ }^{19} \mathrm{~F}-\mathrm{NMR}$ peak areas (left black axis) and $\mathrm{OD}_{330}$ (right gold axis) are plotted as a function of aggregation time. The peak area data points are colored blue for tetramer $(T)$, red for monomer $(\mathrm{M})$, and green for the missing 
signal amplitude for the ensemble of aggregates (A). The green data points are derived by subtracting normalized $\mathrm{T}$ or $\mathrm{M}+\mathrm{T}$ intensity from 1 . The maximal $\mathrm{OD}_{330}$ is normalized to the maximum of the A signal for convenience of comparison. The black lines show the fits of the NMR kinetic data based on the two-step reversible kinetic scheme in (B) at $298 \mathrm{~K}$ and those in (A) at $310 \mathrm{~K}$ are single exponential fits. The orange lines in (A) are single exponential fits to the OD signal. The orange connecting lines in (B) are for eye guidance only. Kinetic data are shown in Table 2.

(1) Wojtczak, A., Cody, V., Luft, J. R., and Pangborn, W. (1996) Structures of human transthyretin complexed with thyroxine at 2.0 A resolution and 3',5'-dinitro- $\mathrm{N}$-acetyl-Lthyronine at 2.2 A resolution, Acta Cryst. D 52, 758-765.

(2) Sun, X., Dyson, H. J., and Wright, P. E. (2018) Kinetic analysis of the multistep aggregation pathway of human transthyretin, Proc. Natl. Acad. Sci. U.S.A. 115, E6201-E6208. 